Pacific Journal of Mathematics

MODEL-COMPLETENESS IN A FIRST ORDER LANGUAGe 


\section{MODEL-COMPLETENESS IN A FIRST ORDER LANGUAGE WITH A GENERALIZED QUANTIFIER}

\section{SHLOMO VINNER}

The concept of Model-Completeness is defined in a first order language with a generalized quantifier. A necessary and sufficient condition is given for that Model-Completeness and its relation to categoricity is discussed.

Some results of this paper were obtained in the author's thesis [12] and were announced in [11]. They, together with other results of [12] were improved independently by the author and by S. Shelah. A suggestion of $S$. Shelah made some proofs simpler and due to it, better results were obtained in Theorem 1.5. The author wishes to thank S. Shelah for his remarks.

Let $L$ be a first order language with equality and let $L(Q)$ be the language obtained from $L$ by adding a new quantifier $Q$. Let $\alpha, \beta$ denote infinite cardinals. We define $\alpha$-satisfaction for $L(Q)$ by interpreting $Q$ as "there exist at least $\alpha$ elements". If a sentence $\phi$ of $L(Q)$ is $\alpha$-satisfied in a model $\mathfrak{A}$ for $L$ we write $\mathfrak{A} k_{\alpha} \phi$ and we say that $\mathfrak{A}$ is an $\alpha$-model for $\phi$. Let $\mathfrak{A}, \mathfrak{B}$ be two models for $L,|\mathfrak{A}| \geqq \alpha$ and $\mathfrak{A} \subseteq \mathfrak{B}$. Write $\mathfrak{A}<_{\alpha} \mathfrak{B}$ if for every $n$, every formula $\phi\left(x_{1}, \cdots, x_{n}\right)$ in $L(Q) \quad$ and every $a_{1}, \cdots, a_{n} \quad$ in $\mathfrak{U}: \mathfrak{U} F_{\alpha} \phi\left[a_{1}, \cdots, a_{n}\right]$ iff $\mathfrak{B} \vDash_{\alpha} \phi\left[a_{1}, \cdots, a_{n}\right]$. Let $T$ be an ordinary first order theory (namely a theory in $L)$ that has infinite models. Define $T(Q)=$ $T \cup\{Q x[x=x]\}$. Call $T \alpha$-model-complete if for every $\mathfrak{H}, \mathfrak{B}$ which are $\alpha$-models for $T(Q)$ and $\mathfrak{U} \subseteq \mathfrak{B}$ also $\mathfrak{U}<_{\alpha} \mathfrak{B}$. A necessary and sufficient condition for $T$ to be $\alpha$-model-complete for $\alpha>\aleph_{0}$ is given in section 1 .

Let $T$ be as before. Define $T(\alpha)=\{\phi: \phi$ is a sentence in $L(Q)$ and for every $\mathfrak{A}$, if $\mathfrak{A} \vdash_{\alpha} T(Q)$ then $\left.\mathfrak{A} \vDash_{\alpha} \phi\right\}$. Call $T \alpha$-complete if for every sentence $\phi$ in $L(Q)$ either $\phi \in T(\alpha)$ or $\neg \phi \in T(\alpha)$. In $\S 2$, it is shown that if $T$ is categorical in one uncountable power, it is $\alpha$ complete and for every $\alpha \geqq \aleph_{0}: T(\alpha)=T\left(\aleph_{0}\right)$. If $T$ is also modelcomplete (in the usual sense) then it is $\alpha$-model-complete for every $\alpha \geqq \aleph_{0}$ and $T(\alpha)$ is decidable provided $T$ is axiomatic.

\section{1. $\alpha$-Model-Completeness.}

Definition 1.1. Let $\phi\left(x, x_{1}, \cdots, x_{m}\right)$ be a formula in $L$ such that $x, x_{1}, \cdots, x_{m}$ are exactly all its free variables. Let $\mathfrak{A}$ be a model for $L$ and let $a_{1}, \cdots, a_{m}$ be elements in $\mathfrak{A}$. Define: 


$$
\phi\left(\mathfrak{A}, a_{1}, \cdots, a_{m}\right)=\left\{a: \mathfrak{U} \vDash \phi\left[a, a_{1}, \cdots, a_{m}\right]\right\} .
$$

Let $T$ be a theory in $L$ and observe the following condition in which $T$ is involved.

Condition 1.1. For every $m \geqq 0$ and every $\phi\left(x, x_{1}, \cdots, x_{m}\right)$ in $L$ there exists an integer $n_{\phi}$ such that for every $\alpha$-model $\mathfrak{A}$ of $T(Q)$ and every $a_{1}, \cdots, a_{m}$ in $\mathfrak{N}$ : if $\left|\phi\left(\mathfrak{A}, a_{1}, \cdots a_{m}\right)\right|>n_{\phi}$ then $\left|\phi\left(\mathfrak{A}, a_{1}, \cdots, a_{m}\right)\right| \geqq$ $\alpha$.

Lemma 1.1. Let $T$ be a theory in $L, \alpha \geqq \mathbb{N}_{0}$. If $T$ fulfills Condition 1.1 then for every formula $\psi$ in $L(Q)$ there exists a formula $\phi$ in $L$ such that $T(Q) \vDash_{\alpha} \psi \leftrightarrow \phi$. (The meaning of the notation " $F_{\alpha}$ " is "semantically valid in $\alpha$ ".)

Proof. Use induction on the structure of $\psi$. The lemma is true for formulae in $L$ and it is clear that if it is true for $\psi, \psi_{1}$ in $L(Q)$ it is also true for $\neg \psi, \psi \wedge \psi_{1}, \exists v \psi$ (for every individual variable $v$ ). We now prove the lemma for $Q v \psi$ assuming it is true for $\psi$. Suppose $\psi$ is $\psi\left(x, x_{1}, \cdots, x_{m}\right)$ and $v$ is $x$. Let $\phi\left(x, x_{1}, \cdots, x_{m}\right)$ be a formula in $L$ such that $T(Q) \vDash_{\alpha} \psi \leftrightarrow \phi$. Let $n$ be an integer the existence of which is assumed in Condition 1.1. Let $\exists^{\geqq n+1} x \phi\left(x, x_{1}, \cdots, x_{n}\right)$ be a formula of $L$ "saying" that there are at least $n+1$ different elements $x$ such that $\phi\left(x, x_{1}, \cdots, x_{m}\right)$ (here we use the assumption that $L$ contains the equality sign). It is easy to see that for every model $\mathfrak{A}$, if $\mathfrak{A}$ is a model of $T(Q)$ and $a_{1}, \cdots, a_{m} \in \mathfrak{U}$ then

$$
\mathfrak{A} \vDash_{\alpha} Q x \psi\left(x, \alpha_{1}, \cdots, a_{m}\right) \leftrightarrow \exists^{\geqq n+1} x \phi\left(x, x_{1}, \cdots, x_{m}\right) .
$$

Hence

$$
T(Q) \vDash{ }_{\alpha} Q x \psi\left(x, x_{1}, \cdots, x_{m}\right) \leftrightarrow \exists^{\geqq n+1} x \phi\left(x, x_{1}, \cdots, x_{m}\right) .
$$

Therefore $\exists^{\geq_{n+1}} x \phi$ is the required formula for $Q x \psi$.

Note that Lemma 1.1 is true also when $L$ is uncountable.

An Example. Let $T$ be the first order theory of a dense linear ordering having neither first nor last element. Using the well known elimination of quantifiers (e.g. Kreisel and Krivine [6]) it is easy to see that $T$ fulfills Condition 1.1 for $\alpha=\aleph_{0}$ but not for $\alpha>\aleph_{0}$.

Now again let $T$ be a theory in $L$ but suppose $\alpha>\aleph_{0}$ and observe the following condition involving $T$.

Condition 1.2. For every $m \geqq 0$, every formula $\phi\left(x, x_{1}, \cdots, x_{m}\right)$ in $L$, every $\alpha$-model $\mathfrak{A}$ of $T(Q)$ and every $a_{1}, \cdots, a_{m} \in \mathfrak{A}$ either $\left|\phi\left(\mathfrak{A}, a_{1}, \cdots, a_{m}\right)\right|<\mathfrak{N}_{0}$ or $\left|\phi\left(\mathfrak{A}, a_{1}, \cdots, a_{m}\right)\right| \geqq \alpha$. 
The following lemma settles the relation between Condition 1.1 and Condition 2.2.

LEMMA 1.2. Let $T$ be a theory in a language L (possibly uncount able). Then for every $\alpha>|L|$ Condition 1.1 is equivalent to Condition 1.2 .

Proof. It is clear that if Condition 1.1 holds, then also Condition 1.2 holds. Choose any cardinal $\mu$ such that $2^{\mu}>|L|$. By Keisler [4] (Theorem 3.3 (iii), p. 121) if $D$ is a regular ultra filter on $\mu$ there exist natural numbers $n_{\nu}, \nu<\mu$, such that $D$-Prod $\lambda_{\nu} n_{\nu}=2^{\mu}$. Suppose that Condition 1.2 holds but Condition 1.1 does not hold. Hence there exists a formula $\phi\left(x, x_{1}, \cdots, x_{m}\right)$ in $L$ such that for every $n_{\nu}, \nu<\mu$, it is possible to find an $\alpha$-model $\mathfrak{A}_{\nu}$ of $T(Q)$ and elements $a_{\nu 1}, \cdots, a_{\nu_{m}}$ in $\mathfrak{A}_{\nu}$ such that $n_{\nu}<\left|\phi\left(\mathfrak{A}_{\nu}, a_{\nu_{1}}, \cdots, a_{\nu_{m}}\right)\right|<\alpha$. Since Condition 1.2 holds we obtain: $\quad n_{\nu}<\left|\phi\left(\mathfrak{H}_{\nu}, a_{\nu 1}, \cdots, a_{\nu_{m}}\right)\right|<\mathcal{N}_{0}$. By Skolem-Lowenheim Theorem we are allowed to suppose that $\left|\mathfrak{A}_{\nu}\right|=2^{2^{\mu, \aleph_{0}}}$ (where $2^{K, 0}=K$, $2^{K, n+1}=2^{2^{K, n}}$ and $2^{K, \aleph_{0}}=\sup \left\{2^{K, n}: n<\mathcal{N}_{0}\right\}$ for every infinite cardinal $K)$. Observe now the structures $\left(\mathfrak{A}_{\nu}, \phi\left(\mathfrak{A}_{\nu}, a_{\nu 1}, \cdots, a_{\nu m}\right)\right)$ and take the ultra product $D$-Prod $\lambda_{\nu}\left(\mathfrak{A}_{\nu}, \phi\left(\mathfrak{A}_{\nu}, a_{\nu}, \cdots, a_{\nu m}\right)\right)$. Denote it by $\left(\mathfrak{B}, \phi\left(\mathfrak{B}, b_{1}, \cdots, b_{m}\right)\right)$. Then:

$$
|\mathfrak{B}| \geqq 2^{2^{\mu}, \mathbf{T}_{0}}>\left|\phi\left(\mathfrak{B}, b_{1}, \cdots, b_{m}\right)\right|=2^{\mu}>|L| .
$$

Therefore we can use Vaught [10] (the generalization of Corollary 4.2, p. 401). Hence, there exists an $\alpha$-model $\sqrt{5}$ of $T(Q)$ and elements $c_{1}, \cdots, c_{m}$ in $\mathbb{S}$ such that $\left|\phi\left(\mathbb{E}, c_{1}, \cdots, c_{m}\right)\right|=\mathcal{N}_{0}$, a contradiction to the assumption that Condition 1.2 holds.

In some applications it is simpler to deal with Condition 1.2 than with Condition 1.1, so there is also a practical purpose in Lemma 1.2.

Lemma 1.3. Let $T$ be any first order theory, $\alpha \geqq \aleph_{0}$. If $T$ is model-complete (in the usual sense) and $T$ fulfills Condition 1.1 then $T$ is $\alpha$-model-complete.

Proof. Use Lemma 1.1.

Lemma 1.4. Let $T$ be a theory in L and suppose $\alpha>|L|$. If $T$ is $\alpha$-model-complete then $T$ fulfills Condition 1.1.

Proof. Suppose that $T$ does not fulfill Condition 1.1. Then by Lemma 1.2 it also does not fulfill Condition 1.2. Therefore there exists an $\alpha$-model $\mathfrak{A}$ of $T(Q)$, a formula $\phi\left(x, x_{1}, \cdots, x_{m}\right)$ in $L$ and elements $a_{1}, \cdots, a_{m}$ in $\mathfrak{A}$ such that $\aleph_{0} \leqq\left|\phi\left(\mathfrak{A}, a_{1}, \cdots, a_{m}\right)\right|<\alpha$. Let $C$ be any set 
of power $\alpha$ such that $C$ and the domain of $\mathfrak{A}$ are disjoint. Denote by $D(\mathfrak{A})$ the diagram of $\mathfrak{A}$ and let $T^{\prime}$ be the following set of sentences:

$T \cup D(\mathfrak{A}) \cup\left\{\phi\left(c, a_{1}, \cdots, a_{m}\right): c \in C\right\} \cup\left\{c_{1} \neq c_{2}\right.$ : for every two different elements $c_{1}, c_{2}$, in $C$.

$T^{\prime}$ is a first order theory and every finite subset of $T^{\prime}$ has a model. Hence, by the Compactness Theorem, $T^{\prime}$ has a model $\mathfrak{A}^{\prime}$. Since $\mathfrak{A} \subseteq \mathfrak{U}^{\prime}$ and $T$ is $\alpha$-model-complete then $\mathfrak{A}<_{\alpha} \mathfrak{A}^{\prime}$. But $\mathfrak{A} \vDash_{\alpha} \neg Q x \phi\left(x, a_{1}, \cdots, a_{m}\right)$ while $\mathfrak{U}^{\prime} \vDash_{\alpha} Q x \phi\left(x, a_{1}, \cdots, a_{m}\right)$, a contradiction.

For a theory $T$ in $L$ such that $\alpha>|L|$ Lemmas 1.3 and 1.4 yield the following:

Theorem 1.5. Let $T$ be a theory in L. Suppose $T$ is modelcomplete (in the usual sense) and $\alpha>|L|$. Then $a$ sufficient and necessary condition for $T$ to be $\alpha$-model-complete is Condition 1.1.

It is possible to look at Theorem 1.5 also from the aspect of definability. Let $\mathfrak{A}$ be a model for $L,|\mathfrak{A}| \geqq \alpha$. Suppose $A_{1} \subseteq$ $\mathfrak{A}$. Call $A_{1} \alpha$-parametrically definable in $\mathfrak{A}$ if there exist a formula $\phi\left(x, x_{1}, \cdots, x_{m}\right)$ in $L(Q)$ and elements $a_{1}, \cdots, a_{m}$ in $\mathfrak{A}$ such that for every $a$ in $\mathfrak{A}, a \in A_{1}$ iff $\mathfrak{U} \vDash_{\alpha} \phi\left(a, a_{1}, \cdots, a_{m}\right)$. By Lemmas 1.1-1.4 we obtain at once:

THEOREM 1.5*. Let $T$ be a theory in $L$ which is also modelcomplete. Suppose $\alpha>|L|$. Then $T$ is $\alpha$-model complete iff for every $\alpha$-model $\mathfrak{A}$ of $T(Q)$ and for every set $A_{1} \subseteq \mathfrak{A}$, if $A_{1}$ is $\alpha$-parametrically definable in $\mathfrak{U}$ then $\left|A_{1}\right|<\aleph_{0}$ or $\left|A_{1}\right| \geqq \alpha$.

We proceed with this section by relating to some known modelcomplete theories. The theory of totally discrete linear ordering having neither first nor last element is model-complete (in the usual sense) but for every $\alpha \geqq \aleph_{0}$ it is not $\alpha$-model-complete.

The theory of dense linear ordering having neither first nor last element is $\boldsymbol{\aleph}_{0}$-model complete but for every $\alpha \geqq \aleph_{1}$ it is not $\alpha$ model complete. For the theory of algebraically closed fields and the theory of real closed fields we have the following theorem:

THEOREM 1.6. Let $T$ be the theory of algebraically closed fields or the theory of real closed fields. Let $\phi\left(x_{1}, \cdots, x_{n}\right)$ be any formula in $L(Q)$ (where $L$ is the language of $T$ ). Then there exists a quantifier free formula $\psi\left(x_{1}, \cdots, x_{n}\right)$ such that $T(Q) F_{\alpha} \phi\left(x_{1}, \cdots, x_{n}\right) \leftrightarrow \psi\left(x_{1}, \cdots, x_{n}\right)$ for every $\alpha \geqq \aleph_{0}$. 
Proof. The proof is similar to the usual elimination of quantifiers for these theories (e.g. Kreisel and Krivine [6]).

COROLlaRy 1.7. The theory of algebraically closed fields and the theory of real closed fields are $\alpha$-model-complete for every $\alpha \geqq \aleph_{0}$.

The last theorem of this section gives a partial answer to a natural question, that is, what conclusions about $\beta$-model-completeness can be made assuming $\alpha$-model-completeness? Using Fuhrken [2] and Keisler [3], one can prove in a straightforward manner that:

THEOREM 1.8. Let $T$ be a countable first order theory.

(1) If $T$ is $\alpha$-model-complete, $\alpha>\aleph_{0}$, then it is also $\aleph_{0}$ model-complete.

(2) If $T$ is $\aleph_{1}$-model-complete, then $T$ is $\alpha$-model-complete for every regular $\alpha$.

(3) (G.C.H) If $T$ is $\alpha$-model-complete where $\alpha$ is a successor of a regular cardinal, then $T$ is $\beta$-model-complete for every regular $\beta$.

(4) If $T$ is $\alpha$-model-complete where $\alpha$ is a singular cardinal, then $T$ is $\beta$-model-complete for every strong limit cardinal $\beta$.

(5) (G.C.H) If $T$ is $\alpha$-model-complete where $\alpha$ is a singular cardinal then $T$ is $\beta$-model-complete for every singular $\beta$.

Proof. All the parts of the theorem are proved in the same way so it will be enough if we prove for example part (1).

Assume that $T$ is $\alpha$-model-complete but not $\aleph_{0}$-model complete. Then there exist two $\aleph_{0}$-models $\mathfrak{A}, \mathfrak{B}$ for $T(Q), \mathfrak{A} \subseteq \mathfrak{B}$, and there exist a formula $\phi\left(x_{1}, \cdots, x_{m}\right)$ in $L(Q)$ and elements $a_{1}, \cdots, a_{m}$ in $\mathfrak{A}$ such that $\mathfrak{A} \vdash_{\mathfrak{T}_{0}} \phi\left[a_{1}, \cdots, a_{m}\right]$ while $\mathfrak{B} \vdash_{\mathfrak{T}_{0}}-\phi\left[a_{1}, \cdots, a_{m}\right]$. By Fuhrken [2] we may assume that $|\mathfrak{A}|=|\mathfrak{B}|=\mathfrak{\aleph}_{0}$. We also may assume for the sake of simplicity that none of the elements $a_{1}, \cdots, a_{m}$ is an interpretation of an individual constant in the language of $T$ and also that this language does not contain functions symbols. Let $c_{1}, \cdots, c_{m}, P(x)$ be $m$ new individual constants and a new unary predicate, respectively. Let $\psi$ be any formula in $L(Q)$. Write $\psi^{P}$ for the formula obtained from $\psi$ by relativizing all the quantifiers of $\psi$ to $P$ (the relativisation of $Q$ is exactly as the relativisation of the existential quantifier). Denote: $T^{P}=\left\{\psi^{P}: \psi \in T\right\}$. Let $S$ be the following set of sentences:

$$
T \cup T^{p} \cup\left\{Q x P(x), \underset{1 \leqq i \leqq m}{\wedge} P\left(c_{i}\right), \phi^{P}\left(c_{1}, \cdots, c_{m}\right), \neg \phi\left(c_{1}, \cdots, c_{m}\right)\right\} .
$$

It is easy to see that a suitable expansion of $\mathfrak{B}$ is an $\boldsymbol{N}_{0}$-model of $S . \quad B y$ Fuhrken [2] it follows that there exists an $\alpha$-model $\mathfrak{D}^{\prime}$ of $S$. Define $C=\left\{d: \mathfrak{D}^{\prime} \vdash P[d]\right\}$. Let $\mathfrak{D}$ be the model obtained from $\mathfrak{D}^{\prime}$ by reducing 
$\mathfrak{D}^{\prime}$ to the language of $T$. Let $\mathbb{S}$ be the submodel of $\mathfrak{D}$ built on $C$ ( $\mathbb{S}$ is a submodel since we assumed that our language does not contain functions symbols). It follows immediately that $\mathfrak{S}, \mathfrak{D} F_{\alpha} T(Q)$. Denote by $d_{1}, \cdots, d_{m}$ the elements of $\mathfrak{D}$ which correspond to the individual constants $c_{1}, \cdots, c_{m}$. Then $\mathcal{C} k_{\alpha} \phi\left[d_{1}, \cdots, d_{m}\right]$, $\mathcal{D} \mathfrak{F}_{\alpha} \neg \phi\left[d_{1}, \cdots, d_{m}\right]$, a contradiction to the assumption that $T$ is $\alpha$-model complete.

A similar theorem, concerning the connections between $\alpha$ completeness and $\beta$-completeness, can be formulated.

It is unknown whether this result is the best result one can obtain.

2. $\alpha$-Completeness, categoricity and $\alpha$-modelcompleteness. Recall now the notions $T(\alpha)$ and $\alpha$-completeness in the beginning. As an analogue to Vaught's Theorem about the connection between categoricity and completeness we have here:

THEOREM 2.1. Let T be a countable first order theory categorical in an uncountable power. Then, for every $\alpha \geqq \aleph_{0}, T$ is $\alpha$-complete and $T(\alpha)=T\left(\kappa_{0}\right)$.

Proof. If $T$ is not $\aleph_{0}$-complete then there exist two $\aleph_{0}$-models $\mathfrak{A}, \mathfrak{B}$ of $T(Q)$ and there exists a sentence $\phi$ in $L(Q)$ such that $\mathfrak{A} F_{\aleph_{0}} \phi$ and $\mathfrak{B} \vdash_{\mathfrak{N}_{0}} \neg \phi$. By Fuhrken [2] there exist two $\mathfrak{N}_{1}$-models $\mathfrak{U}_{1}, \mathfrak{B}_{1}$ such that $\mathfrak{A}_{1} \mathfrak{F}_{\mathbb{N}_{1}} \phi, \mathfrak{B}_{1} \mathfrak{F}_{\boldsymbol{N}_{1}} \neg \phi$ and $\left|\mathfrak{A}_{1}\right|=\left|\mathfrak{B}_{1}\right|=\mathfrak{N}_{1}$. If $T$ is not $\alpha$-complete for $\alpha>\mathfrak{N}_{0}$, then there exist two $\alpha$-models $\mathfrak{A}_{1}, \mathfrak{B}_{1}$ of $T(Q)$ and a sentence $\phi$ in $L(Q)$ such that $\mathfrak{A}_{1} F_{\alpha} \phi, \mathfrak{B}_{1} F_{\alpha} \neg \phi$ and $\left|\mathfrak{A}_{1}\right|=\left|\mathfrak{B}_{1}\right|=\alpha$. So whether $\alpha=\mathcal{N}_{0}$ or $\alpha>\mathcal{N}_{0}$ the assumption that $T$ is not $\alpha$-complete leads us to two uncountable models of $T$ that have the same power and are not isomorphic, a contradiction to Morley [7]. Suppose now that there exists $\alpha$ such that $T(\alpha) \neq T\left(\boldsymbol{N}_{0}\right)$. Since $T$ is $\boldsymbol{\aleph}_{0}$-complete and also $\alpha$-complete there exists $\phi$ in $L(Q)$ such that $\phi \in T\left(\boldsymbol{N}_{0}\right)$ and $\neg \phi \in$ $T(\alpha)$. By Fuhrken [2] there exists an $\alpha$-model $\mathfrak{A}$ for $T(Q)$ such that $\mathfrak{P} F_{\alpha} \phi$, a contradiction to the assumption that $\neg \phi \in T(\alpha)$.

Remark. If $T$ is categorical in $\aleph_{0}$ then $T$ is also $\boldsymbol{N}_{0}$-complete but it is not necessarily $\alpha$-complete for $\alpha>\mathcal{N}_{0}$. One can easily see that by taking $T$ as the theory of dense linear ordering (having neither first nor last element). Again as in the previous section arises the question about the connection between $\alpha$-completeness and $\beta$-completeness and the answer here is the same as there. Another question about $\alpha$ completeness is to find a sufficient and necessary condition on formulae in $L$ so that $T$ will be $\alpha$-complete; but what we know about $\alpha$ completeness are Theorems 2.2 and 2.3.

Let $\phi(x)$ be a formula in $L(Q)$ having $x$ as its only free variable. Let $\mathfrak{A}$ be a model for $L$. Denote $\phi(\mathfrak{A}, \alpha)=\left\{a: \mathfrak{A} \vDash_{\alpha} \phi[a]\right\}$. 
THEOREM 2.2. Let T be a countable first order theory. Assume $T$ is $\alpha$-complete, $\alpha>\aleph_{0}$. Then for every formula $\phi(x)$ in $L(Q)$ (having $x$ as its only free variable) there exists a cardinal $m_{\phi}$, finite or equal to $\alpha$, such that.for every model $\mathfrak{U}$ for $T$ of power $\alpha,|\phi(\mathfrak{A}, \alpha)|=m_{\phi}$.

Proof. Let $\mathfrak{A}$ be any countable model for $T$ (there exists such a model since by the definition of $\alpha$-completeness $T$ has infinite models. It has also a countable model because it is countable). It is clear that either $\mathfrak{A} \vDash_{x_{0}} Q x \phi(x)$ or $\mathfrak{A} \vDash_{N_{0}} \neg Q x \phi(x)$. In the first case define $\Sigma_{1}=T(Q) \cup\{Q x \phi(x)\}$. By Fuhrken [2] there exists an $\alpha$-model $\mathfrak{A}_{1}$ for $\Sigma_{1}$. Since $\mathfrak{A}_{1} \mathcal{F}_{\alpha} Q x \phi(x)$ and $T$ is $\alpha$-complete then for every $\alpha$-model $\mathfrak{A}$ for $T(Q), \mathfrak{A} \vDash_{\alpha} Q x \phi(x)$. Hence $m_{\phi}=\alpha$ in this case. In the second case there exists a finite number $k$ such that $\mathfrak{A} \vdash_{\alpha_{0}} \exists^{k} ! x \phi(x)$, where $\exists^{k} ! x \phi(x)$ is a formula in $L(Q)$ "saying" that there are exactly $k$ elements $x$ such that $\phi(x)$. Define $\Sigma_{2}=T(Q) \cup\left\{\exists^{k} ! x \phi(x)\right\}$. By the same argument as before there exists an $\alpha$-model $\mathfrak{A}_{2}$ for $\Sigma_{2}$. Because of the $\alpha$-completeness of $T$ we obtain $\mathfrak{A} \vDash_{\alpha} \exists^{k} ! x \phi(x)$ for every $\alpha$-model $\mathfrak{A}$ of $T(Q)$. Hence, in this case, $m_{\phi}=k$.

THEOREM 2.3. Let $T$ be a complete theory in $L$ which is also $\alpha$ model-complete, $\alpha \geqq \aleph_{0}$. Then $T$ is also $\alpha$-complete.

Proof. Suppose on the contrary that $T$ is not $\alpha$-complete. Then there exist two $\alpha$-models $\mathfrak{A}, \mathfrak{B}$ for $T(Q)$ and a sentence $\phi$ in $L(Q)$ such that $\mathfrak{A} \vDash_{\alpha} \phi$ and $\mathfrak{B} \vdash_{\alpha} \neg \phi$. Since $T$ is complete then $\mathfrak{A}$ is elementary equivalent to $\mathfrak{B}$. By Bell and Slomson [1] (p. 161), there exists a model $\mathfrak{D}$ which is an elementary extension of $\mathfrak{A}$ and $\mathfrak{B}$. Since $T$ is $\alpha$-modelcomplete and $\mathfrak{A} \mathfrak{F}_{\alpha} \phi$ it follows that $\mathfrak{D} \mathfrak{F}_{\alpha} \phi$. By the same argument we obtain also $\mathfrak{D F}_{\alpha} \neg \phi$, a contradiction.

Definition 2.1. Let $L(Q)$ be recursive and let $T$ be a theory in $L$. Call $T \alpha$-decidable if $T(\alpha)$ is recursive (more precisely, the set of Gödel-Numbers of all the sentences in $T(\alpha)$ is recursive).

THEOREM 2.4. Let T be a theory in L categorical in an uncountable power. Suppose $L(Q)$ and $T$ are recursive. Then $T$ is $\alpha$-decidable for every $\alpha \geqq \aleph_{0}$.

Proof. By Theorem 2.1 we have: $T(\alpha)=T\left(\aleph_{0}\right)$ for every $\alpha \geqq$ $N_{0}$. So it is sufficient to show that $T\left(N_{1}\right)$ is recursive. By Keisler [5] we know that $T\left(\boldsymbol{N}_{1}\right)$ is recursively enumerable. Since $T$ is $\boldsymbol{N}_{1}$-complete then for every $\phi$ in $L(Q), \phi \in T\left(\aleph_{1}\right)$ iff $\neg \phi \notin T\left(\aleph_{1}\right)$. This means that also the complement of $T\left(\aleph_{1}\right)$ is recursively enumerable. Hence $T\left(\aleph_{1}\right)$ is recursive. 
LEMMA 2.5. Let $T$ be any theory in a countable first order language $L$ such that $T$ is categorical in an uncountable power. Let $\mathfrak{A}$ be a model for $T$ and let $a_{1}, \cdots, a_{n}$ be elements in $\mathfrak{A}$. Suppose $|\mathfrak{H}|=\alpha>\mathfrak{N}_{0}$ and $\phi\left(x, x_{1}, \cdots, x_{n}\right)$ is a formula in L having exactly $x, x_{1}, \cdots, x_{n}$ as free variables. Then $\left|\phi\left(\mathfrak{R}, a_{1}, \cdots, a_{n}\right)\right|=\alpha$ or $\left|\phi\left(a, a_{1}, \cdots, z_{n}\right)\right|<\mathfrak{N}_{0}$.

Proof. Since $\alpha>\boldsymbol{N}_{0}$ then $T$ is categorical in $\alpha$, by Morley [7]. Denote by $T\left(\left(\mathfrak{P}, a_{1}, \cdots, a_{n}\right)\right)$ the (first order) theory of $\left(\mathfrak{A}, a_{1}, \cdots, a_{n}\right)$. Again by Morley [7] it is easy to see that $T\left(\left(\mathfrak{A}, a_{1}, \cdots, a_{n}\right)\right)$ is categorical in $\alpha$ so $\left(\mathfrak{A}, a_{1}, \cdots, a_{n}\right)$ is a saturated model. It is well known (see for instance Morley and Vaught [8], Theorem 3.7) that in a saturated model each infinite set defined by a formula (in the language for the model) has the power of the whole model. Hence $\left|\phi\left(\mathfrak{I}, a_{1}, \cdots, a_{n}\right)\right|=\alpha$ or $\left|\phi\left(\mathfrak{I}, a_{1}, \cdots, a_{n}\right)\right|<\boldsymbol{N}_{0}$.

Corollary 2.6. Let $T$ be as in Lemma 2.5. Then for every formula $\phi\left(x_{1}, \cdots, x_{n}\right)$ in $L(Q)$ there exists a formula $\psi\left(x_{1}, \cdots, x_{n}\right)$ in $L$ such that $T(Q) \vDash_{\alpha} \phi\left(x_{1}, \cdots, x_{n}\right) \leftrightarrow \psi\left(x_{1}, \cdots, x_{n}\right)$ for every $\alpha \geqq \mathbf{N}_{0}$.

Proof. By Lemmas 2.5, 1.2, 1.1 and Theorem 2.1.

Corollary 2.6 says that the use of the language $L(Q)$ is dispensible for talking about models of $T$; namely, everything that can be said in $L(Q)$ about elements in a model of $T$ can be said about them in $L$.

THEOREM 2.7. Let T be a theory in a countable first order language $L$ such that $T$ is categorical in an uncountable power and also model-complete (in the usual sense). Then

(1) For every formula $\phi\left(x_{1}, \cdots, x_{n}\right)$ in $L(Q)$ there exist two formulae $\psi_{i}\left(x_{1}, \cdots, x_{n}\right), i=1,2$, in $L, \psi_{1}$ is existential, $\psi_{2}$ is universal and $T(Q) \vDash_{\alpha} \phi\left(x_{1}, \cdots, x_{n}\right) \leftrightarrow \psi\left(x_{1}, \cdots, x_{n}\right)$ for every $\alpha \geqq \aleph_{0}$.

(2) $T$ is $\alpha$-model-complete for every $\alpha \geqq \aleph_{0}$.

(3) If $L(Q)$ and $T$ are recursive then there exists an effective procedure to find $\psi_{i}, i=1,2$, that were mentioned in (1).

Proof. (1) Let $\phi\left(x_{1}, \cdots, x_{n}\right)$ be a formula in $L(Q)$. By Corollary 2.6 there exists a formula $\psi\left(x_{1}, \cdots, x_{n}\right)$ in $L$ such that $T(Q)$ $\vdash_{\alpha} \phi\left(x_{1}, \cdots, x_{n}\right) \leftrightarrow \psi\left(x_{1}, \cdots, x_{n}\right)$ for every $\alpha \geqq \aleph_{0}$. By Robinson [9] (Theorem 3.3.11), since $T$ is model-complete, there exist two formulae $\psi_{1}\left(x_{1}, \cdots, x_{n}\right), \psi_{2}\left(x_{1}, \cdots, x_{n}\right)$ in $L, \psi_{1}$ is existential, $\psi_{2}$ is universal and $T \vdash \psi\left(x_{1}, \cdots, x_{n}\right) \leftrightarrow \psi_{i}\left(x_{1}, \cdots, x_{n}\right), i=1,2$. Therefore

$$
T(Q) \vdash_{\alpha} \phi\left(x_{1}, \cdots, x_{n}\right) \leftrightarrow \psi_{i}\left(x_{1}, \cdots, x_{n}\right),
$$

$i=1,2$, for every $\alpha \geqq \aleph_{0}$. 
(2) By the assumption on $T$ and by Corollary 2.6 $T$ is $\alpha$-modelcomplete for every $\alpha \geqq \aleph_{0}$.

(3) Since $L(Q)$ is recursive there is an effective procedure to count all existential formulae (in $L$ ) that have exactly $x_{1}, \cdots, x_{n}$ as free variables. Let $\psi^{\prime}$ be such a formula. By Theorem 2.4 there is an effective procedure to decide whether $\left[\phi \leftrightarrow \psi^{\prime}\right] \in T\left(\boldsymbol{N}_{1}\right)$ or not. Since there exists an existential formula $\psi_{1}$ such that $\left[\phi \leftrightarrow \psi_{1}\right] \in T\left(\boldsymbol{N}_{1}\right)$ we shall find it after finite number of steps. In the same way we shall find a universal formula $\psi_{2}$ such that $\left[\phi \leftrightarrow \psi_{2}\right] \in T\left(\aleph_{1}\right)$.

\section{REFERENCES}

1. J. L. Bell and A. B. Slomson, Models and Ultraproducts, North-Holland, Amsterdam 1971.

2. E. G. Fuhrken, Languages with added quantifier "there exist at least $\boldsymbol{N}_{c}$ ", In: The Theory of Models, edited by J. Addison, L. Henkin and A. Tarski, North-Holland, Amsterdam, 1965, pp. 121-131.

3. H. J. Keisler, Models with Orderings, In: Logic, Methodology and Philosophy of Science, III, Proceedings of the Third International Congress, Amsterdam, 1967, edited by B. Van Rootselaar and J. F. Staal, North-Holland, Amsterdam, 1968, pp. 35-62.

4. —, A survey of ultraproducts, In: Logic, Methodology and Philosophy of Science, Proceedings of the 1964 International Congress, edited by Y. Bar-Hillel, North-Holland, Amsterdam, 1965, pp. 112-126.

5. Logic with the quantifier "there exist uncountably many", Annals of Math. Logic, 1 (1970), 1-94.

6. G. Kreisel and J. L. Krivine, Elements of Mathematical Logic, North-Holland, Amsterdam 1967.

7. M. D. Morely, Categoricity in Power, Trans. Amer. Math. Soc., 114 (1965), 514-538.

8. M. D. Morely and R. L. Vaught, Homogeneous universal models, Math. Scan., 11 (1962), 37-57.

9. A. Robinson, Introduction to Model Theory and to the Metamathematics of Algebra, North-Holland, Amsterdam, 1967.

10. R. L. Vaught, A. Lowenheim-Skolem Theorem for cardinals far apart. In: The Theory of Models, edited by J. Addison, L. Henkin and A. Tarski, North-Holland, Amsterdam, 1965, pp. $390-401$.

11. S. Vinner, Notices Amer. Math. Soc. 17 (2), p. 456.

12. — Thesis, 1971.

Received September 24, 1973.

The Hebrew University of Jerusalem 




\section{Pacific Journal of Mathematics}

\section{Vol. 56, No. $1 \quad$ November, 1975}

Shimshon A. Amitsur, Central embeddings in semi-simple rings .......... 1

David Marion Arnold and Charles Estep Murley, Abelian groups, A, such

that $\operatorname{Hom}(A,---)$ preserves direct sums of copies of $A \ldots \ldots \ldots .$.

Martin Bartelt, An integral representation for strictly continuous linear

operators ................................... 21

Richard G. Burton, Fractional elements in multiplicative lattices......... 35

James Alan Cochran, Growth estimates for the singular values of

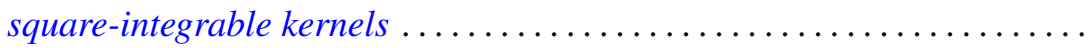

C. Martin Edwards and Peter John Stacey, On group algebras of central

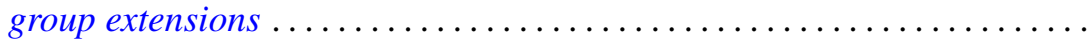

Peter Fletcher and Pei Liu, Topologies compatible with homeomorphism

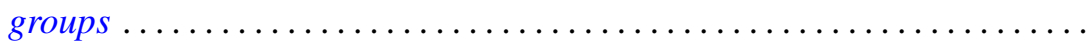

George Gasper, Jr., Products of terminating ${ }_{3} F_{2}(1)$ series ............ 87

Leon Gerber, The orthocentric simplex as an extreme simplex ............

Burrell Washington Helton, A product integral solution of a Riccati

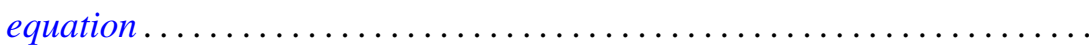

Melvyn W. Jeter, On the extremal elements of the convex cone of



R. H. Johnson, Simple separable graphs .

Margaret Humm Kleinfeld, More on a generalization of commutative and

alternative rings. . .

A. Y. W. Lau, The boundary of a semilattice on an $n$-cell.

Robert F. Lax, The local rigidity of the moduli scheme for curves ...

Glenn Richard Luecke, A note on quasidiagonal and quasitriangular

operators .

Paul Milnes, On the extension of continuous and almost periodic functions

Hidegoro Nakano and Kazumi Nakano, Connector theory.

James Michael Osterburg, Completely outer Galois theory of perfect rings ..................................

Lavon Barry Page, Compact Hankel operators and the F. and M. Riesz

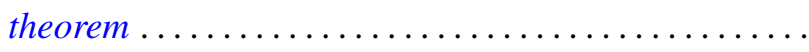

Joseph E. Quinn, Intermediate Riesz spaces..................... 225

Shlomo Vinner, Model-completeness in a first order language with a generalized quantifier.

Jorge Viola-Prioli, On absolutely torsion-free rings ..........

Philip William Walker, A note on differential equations with all solutions of integrable-square............................

Stephen Jeffrey Willson, Equivariant maps between representation 